\title{
Proceso de adaptación de padres y madres de hijos diagnosticados dentro del trastorno del espectro autista
}

\author{
Adaptation process in parents of children \\ diagnosed with Autism Spectrum Disorder

\begin{abstract}
Mariela Sumalavia Casusoㄹ, Carlos A. Almenara²
${ }^{1}$ Facultad de Psicología, Universidad Peruana de Ciencias Aplicadas, Lima, Perú. Email: mariela_sc_@hotmail.com, tel.: (+51) 951025232

${ }^{2}$ Facultad de Psicología, Universidad Peruana de Ciencias Aplicadas, Lima, Perú. Email: carlos.almenara@upc.pe, tel.: (+51 1) 313 33 33, ext. 2803
\end{abstract}

Recibido: 11-06-18

Aceptado: 29-09-18

\section{Resumen}

El presente estudio tuvo como objetivo examinar el proceso de adaptación de padres y madres de hijos diagnosticados dentro del trastorno del espectro autista (TEA). Se utilizó como marco el Modelo Doble ABCX de Adaptación Familiar (McCubbin \& Patterson, 1983). Para esta investigación participaron siete padres de familia (cinco madres y dos padres), quienes tenían un hijo diagnosticado con TEA, el cual se atendía en alguna institución especializada de Lima. Se utilizó el método de investigación cualitativo con un diseño fenomenológico. Asimismo, se realizó una entrevista semiestructurada para la recolección de información y el análisis temático como estrategia de análisis. Los resultados revelaron que los padres de niños con TEA presentaban una serie de sentimientos y actitudes frente al diagnóstico (p. ej. tristeza, frustración, incertidumbre, estrés). Sin embargo, tales sentimientos estaban muy relacionados con las oportunidades de obtener atención profesional apropiada, así como información adecuada respecto al TEA. Asimismo, los participantes destacaron la importancia de fuentes de soporte social. Finalmente, resaltaron la necesidad de una adecuada ayuda profesional para poder afrontar 
las diversas situaciones problemáticas que perciben. Se concluye que el Modelo Doble ABCX de Adaptación Familiar es útil para examinar este proceso. Asimismo, es necesario proveer a la población de información adecuada respecto al TEA, así como de una atención profesional especializada. Ello tiene como fin facilitar un proceso de Adaptación Familiar positivo y, por ende, promover el bienestar general de las personas con trastorno del espectro autista y de sus familias.

Palabras clave: padres, Adaptación Familiar, trastorno del espectro autista, Modelo Doble $A B C X$.

\section{Abstract}

The objective of this study was to examine the adaptation process in parents of children diagnosed with Autism Spectrum Disorder (ASD). The Double ABCX model of family adaptation (McCubbin \& Patterson, 1983) was used in this research. For this investigation, seven parents (five mothers and two fathers) participated. All of them had a son diagnosed with ASD, who was attended in a specialized institution in Lima. A qualitative research method with a phenomenological design was used. In addition, a semi-structured interview was held to gather information, and thematic analysis was used as the analysis strategy. The results revealed parents of children with ASD presented a series of feelings and attitudes towards the diagnosis (e.g., sadness, frustration, uncertainty, stress). However, such feelings were closely related to opportunities to obtain appropriate professional attention and adequate information regarding the ASD. In addition, participants highlighted the necessity for adequate professional help to deal with their problematic situation. In conclusion, the ABCX Double Family Adaptation Model is a useful model to examine this process. Moreover, it is necessary to provide population with adequate information regarding the ASD, as well as specialized professional attention. The purpose of this is to facilitate a process of positive family adaptation, and thus to promote the general well-being of people with autism spectrum disorders and their families.

Keywords: parents, family adaptation, Autism Spectrum Disorder, Double ABCX Model.

La llegada de un hijo suele representar una de las situaciones de mayor felicidad para las parejas, ya que se genera en ambos una serie de expectativas y proyectos a futuro respecto a la dinámica familiar, el desarrollo del niño y el rol que cumplirán ellos en su vida (Navarro-Martinez et al., 2013). Sin embargo, este proyecto de vida puede verse modificado cuando este hijo anhelado presenta alguna característica inesperada o incluso alguna incapacidad que altere los planes futuros iniciales (Dá- 
valos Montalvo, 2015). Dicha situación obliga a reformular las expectativas frente a una nueva realidad, la cual involucra el desarrollo del niño en los distintos aspectos de su vida; y a la vez, se deben plantear las formas en que los padres lograrán adaptarse a este inesperado contexto para brindar un soporte familiar adecuado.

En tal sentido, el presente estudio busca describir la forma en que los padres de hijos diagnosticados dentro del trastorno del espectro autista (TEA) utilizan los recursos, tanto propios como del entorno, para adaptarse a los cambios que genera la crianza de un hijo con TEA. Para ello, este estudio utilizó como marco el Modelo Doble ABCX de Adaptación Familiar (McCubbin \& Patterson, 1983).

\section{El trastorno del espectro autista (TEA)}

El TEA se caracteriza por deficiencias persistentes en la comunicación y en la interacción social, así como por patrones restrictivos de comportamiento, intereses o actividades y por poca flexibilidad de pensamiento (American Psychiatric Association, 2013). Asimismo, los síntomas asociados a este trastorno se manifiestan alrededor del período de desarrollo temprano, causando un deterioro clínico significativo en las áreas social y laboral o en otras áreas importantes del funcionamiento habitual del individuo (American Psychiatric Association, 2013). Este deterioro genera una gran carga para la salud del individuo, la familia e incluso para la salud pública. (Baxter et al., 2015).

Actualmente, la prevalencia estimada del TEA alrededor del mundo es de aproximadamente $1.5 \%$ en los países desarrollados (Lyall et al., 2017). En la población de niños y adolescentes (entre 5 y 17 años de edad), la prevalencia estimada es de $16.1 \%$ (Erskine et al., 2017). Sin embargo, los datos acerca de la carga para la salud y de la prevalencia de personas con TEA en países de bajo y medio ingreso son generalmente limitados (Erskine et al., 2017; Hahler \& Elsabbagh, 2015).

Específicamente en el Perú, de acuerdo al Registro Nacional de la Persona con Discapacidad realizado el año 2016 por el Consejo Nacional para la Integración de la Persona con Discapacidad (Conadis), se han inscrito alrededor de 2809 personas diagnosticadas con TEA, lo cual representa el $1.64 \%$ del total de las personas registradas (Conadis, 2016).

Estudios realizados en nuestro país evidencian un alto nivel de estrés tanto en padres como en madres de niños con TEA al ver que la imagen mental que habían elaborado de su hijo o hija difiere de la realidad (Auad, 2009). Además, otros factores que preocupan a los padres son las pocas iniciativas políticas para difundir a la población información acerca de la salud mental, así como el marcado estigma 
social existente respecto a los trastornos mentales (véase Bojórquez Giraldo et al., 2012). La estigmatización social, manifiesta en este país con matices propios de la cultura, afecta sin duda tanto a los individuos con TEA como a sus familiares. Esto causa gran preocupación, ya que estudios previos sugieren que los padres de niños con TEA perciben la estigmatización social, la cual se suma a la dificultad general de criar a un hijo con TEA (Kinnear, Link, Ballan \& Fischbach, 2016). Además de la estigmatización, los padres de individuos con TEA suelen enfrentarse a niveles más altos de estrés, al divorcio, el desplazamiento geográfico forzado (para lograr la atención en salud mental especializada que necesitan), así como a problemas financieros, en comparación con padres de hijos sin TEA (Bonis, 2016; DePape \& Lindsay, 2015). En suma, el TEA presenta una serie de retos para los padres, lo que los lleva necesariamente hacia un proceso de adaptación.

\section{Modelo Doble ABCX de Adaptación Familiar}

Para muchos padres, el conocer el diagnóstico de un trastorno físico o mental en un hijo puede ser un evento sumamente estresante e incluso, algunas veces, una experiencia traumática (DePape \& Lindsay, 2015; Negri \& Castorina, 2014). Sin embargo, es importante señalar que existen familias que logran adecuarse con éxito a este contexto comúnmente desestabilizador. En este sentido, para examinar y comprender mejor el proceso de adaptación de padres de niños con TEA, se han propuesto distintos modelos (véase Negri \& Castorina, 2014). Entre estos modelos, se destaca por su afinidad con el contexto el Modelo Doble ABCX de Adaptación Familiar (McCubbin \& Patterson, 1983). El Modelo Doble ABCX propone explicar cómo diversos factores se relacionan entre sí e influyen simultáneamente en el proceso de adaptación de los padres de hijos con TEA (Tabla 1). 
Tabla 1.

Modelo Doble ABCX de Adaptación Familiar (McCubbin \& Patterson, 1983)

\begin{tabular}{lll}
\hline Factor & Nombre del factor & Descripción del factor \\
\hline aA & Factor estresante & $\begin{array}{l}\text { Evento que determina el desequilibrio del } \\
\text { contexto y las demandas generadas por la si- } \\
\text { tuación. }\end{array}$ \\
& Apoyos sociales & $\begin{array}{l}\text { Recursos con los que contaba la familia antes } \\
\text { del evento, así como los adaptados a las nue- } \\
\text { vas demandas que este genera. }\end{array}$ \\
cC & Percepción del problema & $\begin{array}{l}\text { Significado que la familia atribuye al evento } \\
\text { estresante, a las demandas que van surgiendo } \\
\text { ya su capacidad para hacerles frente. }\end{array}$ \\
& Estrategias de afrontamiento & $\begin{array}{l}\text { Unión entre los factores bB y cC. Representan } \\
\text { los esfuerzos tanto cognitivos como conduc- } \\
\text { tuales que se emplean para manejar la situa- } \\
\text { ción. }\end{array}$ \\
\hline
\end{tabular}

Cabe mencionar que el Modelo Doble ABCX original ha tenido diversas transformaciones que han dado lugar a otros modelos, tales como el Modelo de Adaptación Familiar y Respuesta a la Adaptación (FAAR); el Modelo de Tipología de Ajuste y Adaptación Familiar; y el Modelo de Resiliencia de Estrés, Ajuste y Adaptación Familiar (véase Negri \& Castorina, 2014). Todos ellos fueron elaborados para explicar de manera general el proceso de adaptación de los padres de hijos con discapacidad. Sin embargo, ya que el presente estudio es específicamente con padres de individuos con TEA, se ha preferido utilizar el Modelo Doble ABCX de Adaptación Familiar porque es el que mejor se ajusta a este contexto familiar específico (Negri \& Castorina, 2014).

Consecuentemente, este estudio tiene como objetivo describir el proceso de adaptación de padres y madres de hijos diagnosticados con el trastorno del espectro autista (TEA) basándose en el Modelo Doble ABCX de Adaptación Familiar de McCubbin \& Patterson (1983). Precisamente, se busca identificar los recursos que los padres reconocen como fuentes de apoyo, las ideas que los padres generaron frente al diagnóstico y las formas en que los padres afrontan este nuevo contexto en sus vidas. 


\section{Método}

Esta investigación sigue un método cualitativo, ya que este es particularmente útil cuando queremos empoderar a los participantes para compartir sus historias y así comprender mejor el contexto en el que acaece el problema (Creswell \& Poth, 2018). Específicamente, se utilizó la aproximación fenomenológica, ya que el foco de estudio estuvo en el contexto de vida de los participantes y, particularmente, sus experiencias (Howitt, 2016). De este modo, por medio de la información proporcionada por los participantes, se espera reconocer las características particulares del contexto y la situación en la que se desenvuelven los padres y sus hijos.

\section{Participantes}

En esta investigación, participaron cinco madres y dos padres, entre 25 y 56 años de edad, con hijos entre los 4 y los 15 años de edad, diagnosticados con trastorno del espectro autista. Todos los participantes provenían de instituciones que brindan apoyo y asesoría a padres de personas con TEA en Lima (Perú).

Como criterios de inclusión para la muestra, se consideró una edad mínima en la que los síntomas del TEA hayan requerido un proceso de adaptación por parte de los padres. Estudios previos sugieren que los 19 meses es la edad en la que los padres reportan la aparición de los primeros síntomas de TEA (Turygin, Matson, Williams \& Belva, 2014). Sin embargo, hay una gran variabilidad en la observación de los síntomas entre los 6 meses y los 3.5 años de edad (Martos-Pérez, 2006). Además, algunos síntomas son difíciles de evaluar antes de los dos años, tales como los patrones del lenguaje (Turygin et al., 2014). Por otro lado, se ha observado que los 27 meses es la media de edad en la que los padres recién recurren a ayuda profesional (Turygin et al., 2014). Por todos estos motivos, se estableció entonces como criterio de participación que la edad de los niños fuera superior a los 3 años.

Asimismo, otro criterio de inclusión de la muestra fue que los padres se encuentren en algún tipo de acompañamiento terapéutico, es decir que cuenten con asesoría e información acerca del trastorno del espectro autista, por lo que podrían describir con mayor precisión el proceso que se encuentran viviendo.

\section{Instrumento}

Se utilizó una entrevista semiestructurada, la cual es considerada una estrategia 'dominante' en la investigación cualitativa, ya que permite evaluar los fenómenos caso por caso, al mismo tiempo que permite conservar sus particularidades (Flick, 2007, p. 10). La entrevista tuvo como base una guía de temas formulados como pre- 
guntas, para ser profundizadas durante la entrevista con los padres. Concretamente, las preguntas abordan los siguientes temas: ideas iniciales de los padres acerca de la paternidad o la maternidad, el proceso de búsqueda e identificación del diagnóstico y los temores que se generan. En otras palabras, el diseño del instrumento estuvo alineado con el objetivo de la investigación (i. e., examinar el proceso de adaptación de padres y madres de hijos diagnosticados con TEA). Para validar esta entrevista semiestructurada, se utilizó el criterio de once jueces expertos en TEA (psicólogos clínicos especialistas en el trabajo con niños y otros profesionales de la salud especialistas en TEA). Estos once jueces analizaron la relación existente entre las preguntas diseñadas para la entrevista y la capacidad de estas para evidenciar que la información obtenida a través de ellas permitiese la comprensión del proceso de adaptación de padres de hijos diagnosticados con TEA. Seguidamente, se realizó una prueba piloto de la entrevista con dos padres para evaluar la capacidad del instrumento para medir las variables y lograr el objetivo de la investigación.

\section{Procedimiento}

En Lima, existen diversas instituciones y asociaciones que brindan apoyo y asesoría a padres de personas con TEA, por lo que se las contactó mediante un correo electrónico y visitas directas y se les presentó el proyecto de investigación enfatizando sus objetivos. Asimismo, se adjuntó un consentimiento informado para los potenciales participantes y que de esta manera autoricen o no su participación voluntaria en la investigación. Además, este documento ayudó a garantizar que los participantes tengan un conocimiento previo de las características y los objetivos generales de la investigación. Por otro lado, todos los participantes fueron informados de los compromisos de confidencialidad y respeto de la privacidad en la presente investigación.

Una vez obtenida la muestra, se realizó una entrevista con cada padre (aproximadamente 50 minutos de duración). Finalmente, las entrevistas fueron transcritas y posteriormente analizadas. Para ello se utilizó el análisis temático (Howitt, 2016), el cual consistió en la sistematización de la información agrupando de acuerdo al Modelo Doble ABCX los temas encontrados. El análisis temático nos permite además reconocer las diferencias y las similitudes entre la información encontrada, así como los eventos y las realidades relacionadas con el contexto en que se desarrolla el problema (Mieles Barrera, Tonon \& Alvarado Salgado, 2012).

\section{Resultados}

A continuación se presentan los principales hallazgos del análisis temático de las entrevistas realizadas. Los resultados se basan en las categorías del Modelo Doble $A B C X$, utilizado en nuestro estudio. 
Tabla 2.

\section{Categorías y ejemplos de los resultados obtenidos}

\begin{tabular}{|c|c|}
\hline Categorías & Ejemplos \\
\hline $\begin{array}{l}\text { Factor aA: } \\
\text { Sentimientos } \\
\text { frente al diag- } \\
\text { nóstico de TEA }\end{array}$ & $\begin{array}{l}\text { - "Estábamos tristes porque en ese momento se te derrumban un } \\
\text { poco los sueños. [...] Soñaba con tener un hijo que tal vez sea lo } \\
\text { que tal vez yo no pude ser". (Javier) } \\
\text { - "Tuve miedo, [...] pensé que mi hijo no iba a ser feliz. ¿Qué había } \\
\text { hecho yo para que le pase esto?". (Catalina) } \\
\text { - "Jamás en mi vida había escuchado la palabra autista y no re- } \\
\text { cuerdo qué me dijo el doctor porque recuerdo que empecé a } \\
\text { llorar". (Lily) }\end{array}$ \\
\hline $\begin{array}{l}\text { Factor bB: } \\
\text { Apoyos sociales }\end{array}$ & $\begin{array}{l}\text { - "Creo que hay mucha ignorancia sobre el tema, [...] los especialis- } \\
\text { tas son los primeros en tener un conocimiento limitado". (Luisa) } \\
\text { - } \quad \text { El dolor hubiera sido menos si lo hubiera podido compartir con } \\
\text { mi pareja, porque hasta ahora no es consciente de la condición } \\
\text { de su hijo". (Lily) } \\
\text { "Acá, con las mamás, he sentido apoyo, he conocido más mamás } \\
\text { como el caso de mi hijo y no me he sentido sola, no sentirme que } \\
\text { soy la única, que hay muchos casos". (Camila) }\end{array}$ \\
\hline $\begin{array}{l}\text { Factor cC: } \\
\text { Percepción del } \\
\text { problema }\end{array}$ & $\begin{array}{l}\text { - "La preocupación de qué va a ser de Melisa; obviamente no siem- } \\
\text { pre vamos a estar nosotros". (Luisa) } \\
\text { - "No sé cómo será su vida, si se casará o no se casará, a todas esas } \\
\text { cosas. A ¿qué pasaría si yo ya no viviera?, ¿qué debo dejarle?". } \\
\text { (Camila) } \\
\text { "Obviamente tiene sus limitaciones. Veo que mejora muchísimo } \\
\text { en su psicomotricidad, entiende todo. [...] Todo eso me da cierta } \\
\text { esperanza, quién sabe y pueda hacer algún tipo de actividad que } \\
\text { no requiera altos coeficientes intelectuales". (Javier) }\end{array}$ \\
\hline $\begin{array}{l}\text { Factor BC: } \\
\text { Estrategias de } \\
\text { afrontamiento }\end{array}$ & $\begin{array}{l}\text { - "Tuve que asumir la situación de que yo podía ayudarla. He teni- } \\
\text { do mucho miedo, pero siempre he pensado que tengo que salir } \\
\text { adelante con ella, que lo mejor que le puedo dar son sus estudios". } \\
\text { (Camila) } \\
\text { - "Más ayudo de lejos, viendo qué hacer y organizándola”. (Luisa) }\end{array}$ \\
\hline
\end{tabular}

Nota. Los nombres asignados a los participantes son ficticios. 


\section{Factor aA: Sentimientos en torno al diagnóstico de trastorno del espectro autista}

En esta primera categoría, se reconoce en todos los participantes que cuando sus hijos son diagnosticados con TEA, aparecen diversos sentimientos y pensamientos; estos están influenciados por la falta de conocimiento de lo que es el TEA y de lo que implica para ellos, como cuidadores, y para sus hijos.

En primer lugar, los padres manifiestan sentimientos de tristeza al conocer que sus hijos presentan un trastorno mental y que esto influye en las expectativas de desarrollo futuro que ellos tenían para sus hijos. Asimismo, los padres manifiestan sentimientos de culpa frente al diagnóstico, además de diversos temores. El principal temor encontrado en los padres se relaciona con la falta de información acerca del trastorno. También demuestran temor respecto a las características mismas del TEA, que en muchas ocasiones no es diagnosticado con precisión por los profesionales de atención en salud primaria. Por otro lado, se encontró que algunos padres atraviesan por un sentimiento de negación frente al diagnóstico, por lo que requieren de un periodo para aceptar la condición que presenta su hijo y lograr asimilar el nuevo contexto, así como los cambios que este conlleva.

\section{Factor bB: Apoyo social}

La mayoría de los padres entrevistados perciben un entorno poco sostenedor frente al contexto que les ha tocado vivir. La falta de recursos y de apoyo del entorno influye en cómo los padres perciben esta experiencia. En primer lugar, los padres buscan en los especialistas (e. g., psicólogos, neurólogos, etc.) la información y las respuestas necesarias para comprender las dificultades que presentan sus hijos, así como para tomar las acciones necesarias a lo largo de su desarrollo. En este contexto, los padres manifiestan encontrarse frente a muchas opciones terapéuticas de distintas características, las cuales pueden percibirse como una fuente de apoyo social. Sin embargo, en algunos casos, los especialistas no llegan a cubrir estas necesidades, por lo que los padres manifiestan cierto malestar frente a la búsqueda de terapias e intervenciones apropiadas para sus hijos.

Asimismo, algunos padres buscan apoyo en sus familias y, principalmente, en sus parejas. En este último caso, los participantes manifiestan que las vivencias y las emociones son similares entre ellos frente a la condición de sus hijos. Sin embargo, en algunos casos, las madres, más que los padres, no logran encontrar el apoyo que buscan en sus parejas para hacer frente a la crianza del niño. En tal sentido, es interesante percatarse de que algunas madres manifiestan recibir más bien el apoyo de otras madres que viven situaciones similares. Estas madres reconocen que esta 
fuente de soporte social es muy útil, ya que logran compartir sus experiencias y sus dudas frente a la crianza de niños con TEA. De hecho, los participantes manifiestan que se han formado en nuestro país muchas redes de madres de niños con TEA, particularmente en centros de intervención para personas con esta condición.

\section{Factor cC: Percepción del problema}

Como se ha mencionado, los padres expresan temores acerca del futuro de sus hijos, especialmente en torno a su desarrollo y el establecimiento de su autonomía durante la etapa adulta. Debido a esta inquietud, los padres refieren una particular preocupación porque sus hijos logren desarrollar las habilidades y las destrezas necesarias para valerse por sí mismos (e. g., autovalimiento). Frente a esto, los padres buscan proveer a sus hijos de las herramientas necesarias para desarrollar sus capacidades y sus habilidades, considerando las limitaciones que se puedan presentar en cada caso. Por otro lado, particularmente en el caso de las familias con más de un hijo, los padres refieren tener la incertidumbre acerca de quién se hará cargo del hijo con TEA durante su vida adulta y qué tanto cuidado necesitará de una tercera persona.

\section{Factor BC: Estrategias de afrontamiento}

En esta categoría, se identifica la forma en que los padres utilizan sus recursos para buscar opciones de mejora frente a la percepción que tienen del problema (Pozo, 2010). Algunos padres en este estudio reconocen que la mejor forma de afrontar las dificultades asociadas a la condición de sus hijos es encontrando la ayuda de especialistas, así como terapias que favorezcan su desarrollo.

De igual manera, se pudo observar también que a algunos padres les cuesta identificar recursos y utilizar estrategias para hacer frente al contexto que están viviendo, mientras que otros participantes sí logran utilizar sus fortalezas y sus recursos individuales para afrontar la situación y brindar apoyo a sus hijos.

Por otro lado, algunos padres indican que se involucran en el proceso terapéutico de sus hijos a través de ejercicios y actividades en casa destinados a potenciar distintas capacidades y habilidades, para ello utilizan información terapéutica de medios como Internet o terapias alternativas. En otros casos, los padres prefieren delegar estas tareas a especialistas y terapeutas.

Finalmente, algunos padres manifestaron que se encuentran en un proceso de búsqueda de recursos para involucrarse más con sus hijos, así como de adaptación a sus características y su condición. Se observó incluso que algunos padres presentaban cierto nivel de negación del diagnóstico, ya que refirieron que les costaba aceptar algunas características del TEA. 


\section{Discusión}

El presente estudio, en una muestra de participantes de Lima (Perú), tuvo como objetivo describir el proceso de adaptación de padres y madres de hijos diagnosticados con TEA. Para ello, se utilizó como marco el Modelo Doble ABCX de Adaptación Familiar de McCubbin \& Patterson (1983). A continuación, se discuten en detalle los resultados en las categorías examinadas.

\section{Sentimientos al recibir el diagnóstico de TEA}

Los resultados sugieren que el nivel de estrés y el impacto frente a la noticia del diagnóstico de TEA en el hijo parecen frustrar las expectativas de crianza y la motivación que los padres tenían respecto al hijo. Estudios previos señalan que los padres experimentan mucho estrés durante los primeros años de vida del niño con TEA, período en el que la búsqueda de un diagnóstico y lo que esto conlleva da lugar a momentos críticos para la familia (Pozo, 2010). Sin embargo, estos niveles de estrés de los padres suelen asociarse a la severidad de los problemas conductuales de los hijos con TEA (Harper, Dyches, Harper, Roper \& South, 2013), así como al proceso de diagnóstico (Reed, Picton, Grainger \& Osborne, 2016). Por ejemplo, un estudio reciente con madres de niños con TEA encontró que el tiempo que transcurre desde que se reporta el problema del hijo hasta la obtención de un diagnóstico es el factor que se correlaciona negativamente con el nivel de estrés parental (Reed et al., 2016).

Investigaciones realizadas en Perú sugieren que luego de las primeras emociones de angustia y culpa frente al diagnóstico ocurriría un proceso de adaptación, por lo que el trastorno pasaría a ser identificado como un reto, pues los padres deben aprender a convivir con las nuevas necesidades que se generan. Sin embargo, aunque los niveles iniciales de angustia, depresión y culpa disminuyen a través del proceso de adaptación, estos siguen presentes a lo largo de la crianza del niño (Auad, 2009; Pérsico, 2011).

Al respecto, como bien señalan Negri \& Castorina (2014), no hay duda de que el diagnóstico de un trastorno del neurodesarrollo conlleva un proceso de adaptación similar en las familias (e. g., en la familia de una persona con discapacidad intelectual). Sin embargo, hay procesos adaptativos distintivos de los familiares de una persona con TEA que reflejan las características únicas del diagnóstico de TEA (Negri \& Castorina, 2014). Así, en el caso específico de los padres de individuos con TEA, un estudio reciente de revisión de la literatura encontró en ellos respuestas emocionales mixtas frente al diagnóstico, incluyendo sentimientos contradictorios tales como alivio y desolación al mismo tiempo, así como culpa y remordimiento 
(DePape \& Lindsay, 2015). Asimismo, se evidencia que algunas madres presentan sentimientos de culpa porque suponen que no cumplieron adecuadamente su rol materno al no haber gestado un hijo sano, por lo que les resulta difícil aceptar el diagnóstico y vincularse con el niño (Auad, 2009).

Esto coincide con nuestros hallazgos al observar que los participantes reportaron una diversidad de sentimientos y pensamientos con relación al diagnóstico. Sin embargo, es importante percatarse de que la mayoría de los participantes asocian el impacto negativo del diagnóstico a la poca información que tenían acerca del trastorno, lo cual se relaciona también con la percepción del problema (factor cC en el Modelo Doble ABCX).

Estos hallazgos son relevantes en nuestra región y particularmente en el Perú, ya que si bien existen algunas iniciativas de información acerca de la salud mental en diversos países (Hernandez, 2013), queda aún mucho por hacer en lo que respecta a la salud mental en nuestro país (Bojórquez Giraldo et al., 2012). Por ejemplo, estudios similares en países de la región, como Ecuador y Venezuela, encuentran que la gran mayoría de los padres de niños con TEA no tenían conocimientos sobre este trastorno antes del diagnóstico (Dávalos Montalvo, 2015; Vergara Tuiran, 2013). Así también, la mayoría de los participantes en esta investigación afirmaron desconocer de qué se trataba el trastorno al recibir el diagnóstico, lo cual generaba diversos sentimientos negativos. Esto es un llamado de atención para buscar una mejora en las políticas públicas peruanas respecto a la difusión de información referente a la salud mental de la población del Perú, y de manera particular respecto al TEA. Si bien en nuestro país existe una ley de protección de personas con trastorno del espectro autista (Ley 30150; Conadis, 2017), es importante que el plan nacional diseñado para estas personas se difunda y se ejecute alcanzando al mayor número de familias posible. Además, se recomienda que estas iniciativas se integren con otras estrategias nacionales de salud general y salud mental, en particular. Esto, con el fin de incrementar el impacto positivo en el bienestar de la población.

Asimismo, es importante que estas iniciativas consideren la importancia de reducir el estigma social y la discriminación asociados a los trastornos mentales (Gronholm, Henderson, Deb \& Thornicroft, 2017; Thornicroft et al., 2016). Para ello, se deben considerar factores contextuales que influyen en el conocimiento y en las actitudes de la población, tales como la cultura, las creencias y el contexto sociodemográfico específico (e. g., Hebert \& Koulouglioti, 2010; Suka, Yamauchi \& Sugimori, 2015; Zuckerman, Lindly \& Sinche, 2016). De igual manera, se recomienda que tales intervenciones utilicen instrumentos apropiados de medición de su efecto en los pacientes (O'Connor, Casey \& Clough, 2014; Wei, McGrath, Hayden \& Kutcher, 2016). Finalmente, en línea con el plan nacional antes mencionado, se 
sugiere considerar el uso de la tecnología para empoderar a la población en el cuidado de su salud (Bond, Merolli \& Ahmed, 2016; Kim \& Xie, 2017; Lagumdzija \& King Swing, 2017; Mitu, 2016).

\section{Búsqueda de soporte social}

Los padres participantes mencionaron que buscan fuentes de apoyo familiar y social. En primer lugar, procuran soporte en el entorno cercano, dentro de sus familias, y es la pareja quien se convierte en una fuente primaria de apoyo para abordar las demandas adicionales de crianza de los niños. Este resultado coincide con estudios previos que sugieren que el recibir soporte de la pareja influye positivamente en su relación y en el proceso de adaptación de ambos padres (García-López, Sarriá, Pozo \& Recio, 2016). Sin embargo, se encontró que algunas madres no identifican a sus cónyuges como una fuente de apoyo en el momento de conocer el diagnóstico, es decir, durante la crisis inicial.

Como se mencionó, el proceso de diagnóstico por el que pasan los padres de niños con TEA puede generar mucho estrés y afectar el funcionamiento psicológico y el bienestar general de los padres. Por ejemplo, una percepción positiva del proceso de diagnóstico estuvo negativamente asociada a niveles de ansiedad y depresión en un estudio de madres de niños con TEA (Reed et al., 2016). Así también, otros estudios sugieren que cuando los padres de niños con TEA observan que son ayudados, decrecen sus niveles de estrés e incrementan sus sentimientos de control, lo que resalta la importancia de las fuentes de soporte social (Pozo, Sarriá \& Méndez, 2006; Siman-Tov \& Kaniel, 2011). De esta manera, el soporte emocional del cónyuge es importante, como bien lo refieren algunos padres en nuestro estudio. En general, la evidencia sugiere que los padres de niños con TEA experimentan una menor satisfacción en su relación de pareja, comparada con padres de niños sin discapacidad (Sim, Cordier, Vaz \& Falkmer, 2016). Si bien algunos matrimonios parecen fortalecerse como resultado del cuidado del niño con TEA, otros se distancian (DePape \& Lindsay, 2015). Esto podría deberse al estrés que conlleva el cuidado de un niño con TEA. Así, estudios previos sugieren que a mayor estrés por el cuidado de un hijo con TEA menor será la calidad de la relación marital (Harper et al., 2013). Coincidiendo con ello, otros estudios sugieren que la satisfacción marital podría agravarse también por el nivel de los problemas conductuales del hijo con TEA (Langley, Totsika \& Hastings, 2017). Posteriormente, este impacto negativo en la calidad marital puede afectar el ajuste psicológico, como lo sugieren algunos estudios en colaboración con madres de niños diagnosticados con este trastorno (e. g., Benson \& Kersh, 2011). En suma, esto podría explicar por qué algunas madres en nuestro estudio perciben que sus cónyuges no son una fuente de soporte emocional. En todo caso, estudios predictivos sugieren que el sentido de coherencia, el locus de control interno, el 
soporte social y la calidad del matrimonio incrementan la habilidad para afrontar el estrés que conlleva criar un hijo con TEA (Siman-Tov \& Kaniel, 2011). Luego, se ha visto que un afrontamiento activo, positivo y focalizado en el problema se asocia a un proceso de adaptación más positivo en padres de niños con TEA (Pozo, Sarriá \& Brioso, 2014). Por tales motivos, es importante promover los recursos individuales de los padres de niños con TEA, particularmente de las madres.

Por otro lado, los participantes indican que existe la necesidad de fomentar una conciencia social respecto a la inclusión y la aceptación de personas con discapacidad. Además de ello, los participantes mencionan la necesidad de contar con profesionales especialistas en TEA que puedan ayudar de forma integral en el desarrollo y el bienestar de las personas con este trastorno. Como se señaló anteriormente, los padres de niños con TEA perciben la estigmatización social, que se suma a la dificultad general de criar a un hijo (Kinnear et al., 2016). En el caso particular del Perú, este fenómeno de estigmatización social se ve agravado por una inapropiada atención profesional de la salud mental, como lo refieren los padres y también estudios previos (Bojórquez Giraldo et al., 2012). En otras palabras, como se mencionó anteriormente, es importante la difusión de información continua en la población acerca de temas de salud mental para eliminar creencias erróneas y reducir la estigmatización (Hernandez, 2013).

Sin embargo, también encontramos participantes que reconocen y valoran el apoyo que reciben de ciertas instituciones, así como el hecho de que a través de ellas conocen a otros padres y madres en situaciones similares. Estas interacciones les permiten compartir experiencias, satisfacciones y temores, así como generar una red de soporte social. Estos hallazgos revelan también la necesidad de diseñar y divulgar información adecuada acerca de los familiares de personas con TEA para que puedan apoyarse entre sí. La evidencia sugiere que estas redes de apoyo social de padres de niños con TEA ayudan a reducir los síntomas de depresión, ansiedad e ira de los padres, y por ende favorecen su calidad de vida y su bienestar (Boyd, 2002; Pozo, 2010). En suma, se sugiere la promoción de las redes de apoyo social para padres de niños con TEA, y que así puedan tener espacios de escucha y de interacción con personas que tienen vivencias similares.

\section{Percepción del problema: Expectativas y temores frente al futuro}

Los padres indican tener una gran preocupación respecto al futuro de sus hijos y muchos de ellos se atemorizan porque este es incierto tanto para sus hijos como para ellos mismos. Muestran como preocupaciones principales el nivel de autonomía del hijo, su desarrollo laboral, su independencia económica, etc. Estudios previos sugieren que un conocimiento adecuado del TEA, así como el apoyo social 
que los padres puedan recibir, generan menos estrés y más percepción de control de la situación (Siman-Tov \& Kaniel, 2011). Esto revela nuevamente la importancia de la concientización y la sensibilización de la población respecto a la salud mental, así como la necesidad de continuar promoviendo las políticas de inclusión que favorecen a las personas con TEA. Asimismo, es importante desarrollar programas de atención en salud física y mental que permitan un cuidado apropiado de las personas con TEA a lo largo de su desarrollo, incluyendo la vida adulta. Esto se sustenta en que la sintomatología del TEA tiende a ser estable a lo largo del desarrollo (Bieleninik et al., 2017), por ello se sugiere que las intervenciones se centren más en la calidad de vida y en el funcionamiento general de las personas con TEA en lugar de focalizarse en los síntomas (Bieleninik et al., 2017). Este soporte profesional debe también promover la aceptación en los padres de niños con TEA (Dardas \& Ahmad, 2015).

\section{Estrategias de afrontamiento}

Los padres participantes señalaron que buscan diversos recursos para afrontar el diagnóstico y sus características, tales como la búsqueda de ayuda profesional que promueva el desarrollo de sus hijos. Por otro lado, algunos padres refirieron haber realizado cambios en el ámbito personal, tales como una mejora en su nivel de tolerancia. Asimismo, muchos padres buscaron informarse sobre el TEA, y alcanzaron un nivel de conocimiento que les permite compartir experiencias con sus hijos y así participar en su desarrollo. Estos hallazgos revelan la importancia de los recursos a los que los padres de niños con TEA pueden acceder para afrontar los diversos problemas que ellos perciben. Como se mencionó, en estudios previos con padres de niños con TEA se observó que el uso de tales estrategias de afrontamiento se asocia con un mejor proceso de adaptación de los padres (Pozo et al., 2014). En tal sentido, la ayuda profesional es muy importante para promover esos recursos individuales. Por tal motivo, se ha venido sugiriendo que los profesionales de la salud brinden apoyo formal y adecuado a los padres de niños con TEA (Dardas \& Ahmad, 2015).

\section{Limitaciones}

Si bien el presente estudio tiene fortalezas, como el uso del Modelo ABCX que cuenta con respaldo empírico, existen limitaciones que debemos señalar. En primer lugar, fue difícil obtener la participación de padres de niños con TEA; el principal motivo fue que muchos de los centros especializados en TEA en este país son privados y, por ende, tienen muchas restricciones de acceso. Esto, como consecuencia, limita la posibilidad de aplicar los resultados al resto de la población de padres de niños con TEA. Por otro lado, son escasos los estudios sobre el TEA en el Perú, particularmente aquellos que puedan ser utilizados como antecedentes. Sin 
embargo, los resultados fueron muy similares a los de estudios internacionales y aportan evidencia al Modelo Doble ABCX.

\section{Conclusiones}

Los resultados sugieren que el Modelo Doble ABCX de Adaptación Familiar es útil para explorar el proceso de adaptación de los padres de niños con trastorno del espectro autista. Por otro lado, los hallazgos revelan la importancia de proveer a la población información adecuada respecto al espectro autista, así como una atención profesional especializada. Todo ello, con el fin general de facilitar un proceso de adaptación familiar positivo y, por ende, promover el bienestar general de las personas con trastorno del espectro autista y sus familias.

\section{Referencias}

American Psychiatric Association. (2013). Diagnostic and statistical manual of mental disorders: DSM-5 (5. ${ }^{a}$ ed.). Arlington, VA: American Psychiatric Association.

Auad, C. M. (2009). Representaciones mentales de la maternidad en madres de hijos con trastorno del espectro autista. Tesis para optar el título de licenciada en Psicología con mención en Psicología Clínica.

Baxter, A. J., Brugha, T. S., Erskine, H. E., Scheurer, R. W., Vos, T., \& Scott, J. G. (2015). The epidemiology and global burden of autism spectrum disorders. Psychological Medicine, 45(3), 601-613. doi: 10.1017/So03329171400172X

Benson, P. R., \& Kersh, J. (2011). Marital quality and psychological adjustment among mothers of children with ASD: Cross-sectional and longitudinal relationships. Journal of Autism and Developmental Disorders, 41(12), 1675-1685. doi: 10.1007/s10803-011-1198-9

Bieleninik, Ł., Posserud, M. B., Geretsegger, M., Thompson, G., Elefant, C., \& Gold, C. (2017). Tracing the temporal stability of autism spectrum diagnosis and severity as measured by the Autism Diagnostic Observation Schedule: A systematic review and meta-analysis. Plos One, 12(9), 1-23. doi: 10.1371/journal. pone.0183160

Bojórquez Giraldo, E., Chacón Antezana, Ó., Rivera Ramírez, G., Donadío, G., Stucchi Portocarrero, S., Sihuas Meza, C., ...María Llanos, A. (2012). Colegio Médico del Perú: Propuesta de reforma de la salud mental en el Perú. Acta Médica Peruana, 29, 43-55.

Bond, C. S., Merolli, M., \& Ahmed, O. H. (2016). Patient empowerment through social media. En S. Syed-Abdul, E. Gabarron, \& A. Y. S. Lau (Eds.), Participatory health through social media (pp. 10-26). London: Elsevier. 
Bonis, S. (2016). Stress and parents of children with autism: A review of literature. Issues in Mental Health Nursing, 37(3), 153-163. doi:10.3109/01612840.2015.1116030

Boyd, B. A. (2002). Examining the relationship between stress and lack of social support in mothers of children with autism. Focus on Autism and Other Developmental Disabilities, 17(4), 208-215. doi: 10.1177/10883576020170040301

CONADIS. (2017). Plan nacional para las personas con Trastorno del Espectro Autista (TEA): 2017-2021. Lima, Perú. Recuperado de: http://www.conadisperu. gob.pe/wp-content/uploads/2017/o4/PLAN-TEA.doc

Creswell, J. W., \& Poth, C. N. (2018). Qualitative inquiry and research design: Choosing among five approaches (4.를. ed.). Thousand Oaks, CA: Sage.

Dardas, L. A., \& Ahmad, M. M. (2015). Coping strategies as mediators and moderators between stress and quality of life among parents of children with autistic disorder. Stress and Health, 31(1), 5-12. doi: 10.1002/smi.2513

Dávalos Montalvo, M. B. (2015). Miedos, mitos y cambios que ocurren en los padres con hijos diagnosticados con Trastorno del Espectro Autista. Universidad de las Américas, Quito. Recuperado de http://dspace.udla.edu.ec/handle/3300o/3448

DePape, A. M., \& Lindsay, S. (2015). Parents' experiences of caring for a child with autism spectrum disorder. Qualitative Health Research, 25(4), 569-583. doi: 10.1177/1049732314552455

Erskine, H. E., Baxter, A. J., Patton, G., Moffitt, T. E., Patel, V., Whiteford, H. A., \& Scott, J. G. (2017). The global coverage of prevalence data for mental disorders in children and adolescents. Epidemiology and Psychiatric Sciences, 26(4), 395-402. doi: 10.1017/S2045796015001158

Flick, U. (2007). Designing qualitative research. Thousand Oaks, CA: Sage.

García-López, C., Sarriá, E., Pozo, P., \& Recio, P. (2016). Supportive dyadic coping and psychological adaptation in couples parenting children with Autism Spectrum Disorder: The role of relationship satisfaction. Journal of Autism and Developmental Disorders, 46(11), 3434-3447. doi: 10.1007/s10803-016-2883-5

Gronholm, P. C., Henderson, C., Deb, T., \& Thornicroft, G. (2017). Interventions to reduce discrimination and stigma: the state of the art. Social Psychiatry and Psychiatric Epidemiology, 52(3), 249-258. doi: 10.1007/s00127-017-1341-9

Hahler, E. M., \& Elsabbagh, M. (2015). Autism: A global perspective. Current Developmental Disorders Reports, 2(1), 58-64. doi: 10.1007/s40474-014-0033-3

Harper, A., Dyches, T. T., Harper, J., Roper, S. O., \& South, M. (2013). Respite care, marital quality, and stress in parents of children with autism spectrum disorders. Journal of Autism and Developmental Disorders, 43(11), 2604-2616. doi: 10.1007/s10803-013-1812-0 
Hebert, E. B., \& Koulouglioti, C. (2010). Parental beliefs about cause and course of their child's autism and outcomes of their beliefs: A review of the literature. Issues in Comprehensive Pediatric Nursing, 33(3), 149-163. doi: 10.3109/01460862.2010.498331

Hernandez, L. M. (2013). Health literacy: Improving health, health systems, and health policy around the world (workshop summary). Washington, D. C.: National Academic Press.

Howitt, D. (2016). Introduction to qualitative research methods in psychology ( $3 .{ }^{\mathrm{a}}$ ed.). Harlow: Pearson.

Kim, H., \& Xie, B. (2017). Health literacy in the eHealth era: A systematic review of the literature. Patient Education and Counseling, 100(6), 1073-1082. doi: 10.1016/j.pec.2017.01.015

Kinnear, S. H., Link, B. G., Ballan, M. S., \& Fischbach, R. L. (2016). Understanding the experience of stigma for parents of children with autism spectrum disorder and the role stigma plays in families' lives. Journal of Autism and Developmental Disorders, 46(3), 942-953. doi:10.1007/s10803-015-2637-9

Lagumdzija, A., \& King Swing, V. (2017). Health, digitalization, and individual empowerment. En F. S. Topor (Ed.), Handbook of research on individualism and identity in the globalized digital age (pp. 380-402). Hershey, PA: IGI Global.

Langley, E., Totsika, V., \& Hastings, R. P. (2017). Parental relationship satisfaction in families of children with autism spectrum disorder (ASD): A multilevel analysis. Autism Research, 10(7), 1259-1268. doi: 10.1002/aur.1773

Lyall, K., Croen, L., Daniels, J., Fallin, M. D., Ladd-Acosta, C., Lee, B. K., ...Newschaffer, C. (2017). The changing epidemiology of autism spectrum disorders. Annual Review of Public Health, 38(1), 81-102. doi: 10.1146/annurev-publhealth-031816-044318

Martos-Pérez, J. (2006). Autismo, neurodesarrollo y detección temprana. Revista de Neurología, 42 (Suppl.), S99-S101.

McCubbin, H. I., \& Patterson, J. M. (1983). The family stress process: The Double ABCX Model of adjustment and adaptation. Marriage $\mathcal{E}$ Family Review, 6(12), 7-37. doi: 10.1300/Joo2vo6no1_02

Mieles Barrera, M., Tonon, G., \& Alvarado Salgado, S. (2012). Investigación cualitativa: El análisis temático para el tratamiento de la información desde el enfoque de la fenomenología social. Universitas Humanística, 74, 195-225.

Mitu, B. (2016). Health in the digital era: Searching health information online. En V. Marinescu \& B. Mitu (Eds.), The power of the media in health communication (pp. 145-156). New York, NY: Routledge.

Navarro-Martinez, E., Valadez-Sierra, M. de los D., Soltero-Rodríguez, N., Zambrano-Guzmán, R., Nava-Bustos, G., \& Sandoval-Martínez, J. (2013). Programa 
de entrenamiento cognitivo conductual para padres de niños con trastorno del espectro autista. Revista de Educación y Desarrollo, 25(abril-junio), 71-76.

Negri, L. M., \& Castorina, L. L. (2014). Family adaptation to a diagnosis of autism spectrum disorder. En J. Tarbox, D. R. Dixon, P. Sturmey, \& J. L. Matson (Eds.), Handbook of early intervention for autism spectrum disorders: research, policy, and practice (pp. 117-137). New York, NY: Springer.

O'Connor, M., Casey, L., \& Clough, B. (2014). Measuring mental health literacy-a review of scale-based measures. Journal of Mental Health, 23(4), 197-204. doi: $10.3109 / 09638237.2014 .910646$

Pérsico, A. F. (2011). Representaciones mentales del autismo en padres de niños diagnosticados con este trastorno. Tesis para optar el título de licenciada en Psicología con mención en Psicología Clínica.

Pozo, P. (2010). Adaptación psicológica en madres y padres de personas con Trastorno del Espectro Autista: Un estudio multidimensional. Universidad Nacional de Educación a Distancia. Recuperado de http://www.tdx.cat/handle/10803/63488

Pozo, P., Sarriá, E., \& Brioso, A. (2014). Family quality of life and psychological wellbeing in parents of children with autism spectrum disorders: A double ABCX model. Journal of Intellectual Disability Research, 58(5), 442-458. doi: 10.1111/ jir.12042

Pozo, P., Sarriá, E., \& Méndez, L. (2006). Estrés en madres de personas con trastornos del espectro autista. Psicothema2, 18(3), 342-347.

Reed, P., Picton, L., Grainger, N., \& Osborne, L. A. (2016). Impact of diagnostic practices on the self-reported health of mothers of recently diagnosed children with ASD. International Journal of Environmental Research and Public Health, 13(9), 888. doi: 10.3390/ijerph13090888

Sim, A., Cordier, R., Vaz, S., \& Falkmer, T. (2016). Relationship satisfaction in couples raising a child with autism spectrum disorder: A systematic review of the literature. Research in Autism Spectrum Disorders, 31, 30-52. doi: 10.1016/j. rasd.2016.07.004

Siman-Tov, A., \& Kaniel, S. (2011). Stress and personal resource as predictors of the adjustment of parents to autistic children: A multivariate model. Journal of Autism and Developmental Disorders, 41(7), 879-89o. doi: 10.1007/s10803o10-1112-X

Suka, M., Yamauchi, T., \& Sugimori, H. (2015). Relationship between individual characteristics, neighbourhood contexts and help-seeking intentions for mental illness. BMJ Open, 5(8). doi: 10.1136/bmjopen-2015-008261

Thornicroft, G., Mehta, N., Clement, S., Evans-Lacko, S., Doherty, M., Rose, D., 
...Henderson, C. (2016). Evidence for effective interventions to reduce mental-health-related stigma and discrimination. The Lancet, 387, 1123-1132. doi: 10.1016/So140-6736(15)00298-6

Turygin, N., Matson, J. L., Williams, L. W., \& Belva, B. C. (2014). The relationship of parental first concerns and autism spectrum disorder in an early intervention sample. Research in Autism Spectrum Disorders, 8(2), 53-60. doi: 10.1016/j. rasd.2013.10.008

Vergara Tuiran, L. M. (2013). Grado de conocimiento de los padres de niños con autismo sobre los conceptos básicos de la enfermedad y los cuidados que requiere. Universidad del Zulia, Venezuela. Recuperado de http://tesis.luz.edu. ve/tde_arquivos/152/TDE-2014-07-16T12:50:19Z-5184/Publico/vergara_tuiran_lorena_marcela.pdf

Wei, Y., McGrath, P. J., Hayden, J., \& Kutcher, S. (2016). Measurement properties of tools measuring mental health knowledge: A systematic review. BMC Psychiatry, 16(1), 297. doi: 10.1186/s12888-016-1012-5

Zuckerman, K. E., Lindly, O. J., \& Sinche, B. (2016). Parent beliefs about the causes of learning and developmental problems among children with autism spectrum disorder: Results from a national survey. American Journal on Intellectual and Developmental Disabilities, 121(5), 432-447. doi: 10.1352/1944-7558121.5 .432 